Military Technical College, Kobry El-Kobbah, Cairo, Egypt

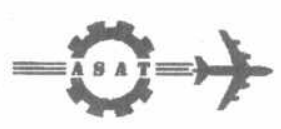

$9^{\text {th }}$ International Conference

On Aerospace Sciences \& Aviation Technology

\title{
WADGPS PROCESSING LATENCY REDUCTION
}

\author{
M. A. Matar" and S. Y. Issa**
}

\section{ABSTRACT}

Global Positioning System (GPS), the most advanced navigation system, suffers three problems that are very pronounced for safety critical applications like Aviation. These problems are namely: accuracy, availability and integrity. Standalone solutions to these problems have been developed and conceptually integrated in a framework known as Satellite Based Augmentation System (SBAS) in the 1990s. This work deals with the GPS positioning accuracy improvement obtainable out of SBAS through reduction of orbit and clock errors. More specifically, implementation of Wide Area Master Station (WMS) covering estimation techniques for these errors is extensively studied on the bases of Kinematic, and Nondynamic approaches. Published approaches rely on solving the problem of orbit error estimation on the bases of transmitted pseudorange errors yielding WMS processing latency of about 7 minutes using P-III $500 \mathrm{MHz}$ IBM PC and a MATLAB 5.3 environment. This processing latency is intolerable and falls very far from the FAA time-to-alarm requirement (about $8 \mathrm{sec}$ for en-route through non-precision approach phases and $5.2 \mathrm{sec}$ for precision approach). To reduce the processing time to the level required by the FAA time-to-alarm, this work proposes to solve an equivalent problem of orbit determination rather than orbit error determination. Research for accelerating and improving the performance of Nondynamic approach has resulted in reducing processing time to about one fiftieth of the corresponding time obtained by implementing published solutions.

\section{KEYWORDS}

Global Positioning System (GPS), Satellite Based Augmentation Systems (SBAS), Wide area differential GPS (WADGPS), satellite ephemeris, orbit, pseudorange, Least squares, processing latency, Wide Area Augmentation Systems (WAAS), Wide Area Master Station (WMS), Wide Area Reference Station (WRS)

\footnotetext{
* Avionics Depart., MTC

** Syrian Assad Academy
} 


\section{INTRODUCTION}

Around the world, several SBAS versions are under development [1-3]. Wide Area Augmentation System (WAAS) is being developed by the FAA, and is expected to provide complete service over the United States and Canada by 2006 [4]. In parallel, Europe is developing the European Geostationary Navigation Overly Service (EGNOS) [5] over the European continent while Japan is developing the MTSAT Satellite-Based Augmentation System (MSAS) [6] for the Eastern coast of Asia. The MSAS and WAAS service areas are too far to cover the Middle East or Arab Region $(A R)$, whereas EGNOS service area with some extension would include that region [13]. To cover this region and some of the neighbor countries, the Arab Wide Area Augmentation System (ARWAAS) has been proposed [12]

SBAS is proposed mainly for enhancement of GPS performance in order to meet the avionics user's requirements from the viewpoints of accuracy, availability and integrity. SBAS is going to improve: the accuracy, by implementing a Wide Area Differential GPS (WADGPS), the availability, by additional Geostationary satellites emitting GPS-like ranging signals and the integrity, by affording GPS Integrity Channel (GIC) that broadcasts satellite health information.

Analysis of the GPS error sources and their contributions reveals that the ephemeris errors, satellite clock error and ionospheric delay error are dominating other sources. To determine their share in positioning errors, a timely coordinated spatial sampling of the ionosphere and satellites orbits is required. From spatial sampling of the ionosphere, reconstruction of the whole ionosphere profile may be found and from sampling of satellite orbits reconstruction of the corresponding orbits and consequently ephemeris correction would be generated. This spatial sampling requires a network that includes at least one Wide Master Station (WMS), a number of the Wide Reference Stations (WRS's), and communication links. Each WRS is equipped with a high-quality clock source and a high-quality GPS receiver capable of tracking satellites within its field of view, a meteorological sensor, a processor, and network hardware for transmission of GPS measurements data to WMS. Within the WMS such data are fused to solve for the GPS error components on the basis of the known WRS locations and the collected information. The computed error corrections are transmitted to the users via Geostationary Satellite or any convenient communication link.

In general, three basic techniques could be used in the WMS to estimate the satellite ephemeris and clock error; namely Nondynamic, Kinematic, and Dynamic techniques. The Nondynamic technique based upon inverted form of the navigation fix solution yields the instantaneous position of the satellite, while Kinematic technique uses velocity information along with a Kalman filter to propagate data over time so as to estimate the position errors. Finally, dynamic technique [11] computes precise satellite orbits from collection of measurements that are related to the satellite states by precise dynamical models accurately describing the orbit motion of the satellite. Due to limited computational resources, the Nondynamic technique will be implemented and extended to Kinematic technique [12].

The present work deals with the "coordinated "WRS-and-WMS implementation issues along with their relevant assessment using the developed algorithms and the satellite tool kit (STK) data. Performance indices are: the WMS processing latency and arithmetic precision of the WRS -to- WMS transmitted data along with their impact on implementation complexity. Section 2 deals with the WRS implementation 
while transmitting either pseudorange or pseudorange errors. Section 3 deals with the WMS implementation of processing algorithms for received data of either pseudorange or pseudorange errors. Results along with their analysis are given in section 4 . Section 5 contains conclusions and suggestions for future work.

\section{WIDE AREA REFERENCE STATION (WRS)}

WRS comprises three independent pieces of equipment, consisting of an antenna GPS receiver (dual-frequency/at least codeless), processor, power conditioner, and frequency standard, in order to provide redundancy and overall system integrity. WRS acquires code observation at the GPS frequencies $f_{\mathrm{L} 1}$ and $f_{\mathrm{L} 2}$ (see Fig.1). The set of code observations (from $\mathrm{K}_{\mathrm{m}}$ satellites in view of the $m^{\text {th }}$ WRS) at $f_{\mathrm{L} 1}$ and $f_{\mathrm{L} 2}$ yields $\left\{\rho_{m, L 1}^{(k)}\right\}_{k=1}^{K_{m}}$ and $\left\{\rho_{m, L 2}^{(k)}\right\}_{k=1}^{K_{m}}$ respectively as:

$$
\begin{aligned}
& \rho_{m, L_{1}}^{(k)}=\left(r_{m}^{(k)}+b_{m}-B^{(k)}\right)+I_{m, L_{1}}^{(k)}+T_{m}^{(k)}+v_{m, \tau}^{(k)} . \\
& \rho_{m, L_{2}}^{(k)}=\left(r_{m}^{(k)}+b_{m}-B^{(k)}\right)+I_{m, L_{2}}^{(k)}+T_{m}^{(k)}+v_{m, \tau}^{(k)} .
\end{aligned}
$$

where ( all quantities are expressed in meters)
$\rho_{m}{ }^{k}$
physical distance between station $\mathrm{m}$ and satellite $\mathrm{k}$.
$B^{k}$ error due to the $m$ station clock offset .
$\mathrm{I}_{\mathrm{m}, \mathrm{L}_{1}}^{\mathrm{k}}, \mathrm{I}_{\mathrm{m}, \mathrm{L}_{2}}^{\mathrm{k}}$ : error due to the $\mathrm{k}$ satellite clock offset. $T_{m}{ }^{k}$ error due to ionospheric time delay between station and satellite $k$ by using the $L_{1}$ and $L 2$ frequencies respectively
$v_{\mathrm{m}, \tau}^{\mathrm{k}}:$ error due to tropospheric error between station $\mathrm{m}$ and satellite $\mathrm{k}$ error due to pseudorange noise

Carrier-smoothing algorithm delivers smoothed pseudorange estimates $\left\{\rho_{m}^{k}\right\}_{k=1}^{K_{m}}$ (free of ionospheric delays) and the ionospheric delay estimates $\left\{I_{m}^{k}\right\}_{k=1}^{K_{m}}$.

The smoothed pseudorange estimates are given by:

$$
\rho_{m}^{(k)}=\left(r_{m}^{(k)}+b_{m}-B^{(k)}\right)+\Delta I_{m}^{(k)}+T_{m}^{(k)}+v_{m, \tau}^{(k)} .
$$

If the ionospheric error term is included in the noise term, this formula reduces to

$$
\rho_{m}^{(k)} \approx\left(r_{m}^{(k)}+b_{m}-B^{(k)}\right)+T_{m}^{(k)}+v_{m, r}^{(k)} .
$$

Computed from the satellite data in the navigation message, the pseudorange and the nominal range to the satellite (known location of the WRS) are compared to get the required correction at the WRS site. The satellite clock offset (described by the clock field in the navigation message) is also taken into account. Thus pseudorange errors reduce to:

$$
\Delta \rho_{m}^{(k)}=\left|r_{m}^{(k)}\right|-\left|r_{m}^{(k, B)}\right|+b_{m}-\left(B^{(k)}-B^{(k, B)}\right)+T_{m}^{(k)}+v_{m, \tau}^{(k)} .
$$




$$
\begin{aligned}
& =\Delta r^{k} \cdot 1_{m}^{k}+b_{m}-\left(B^{k}-B^{k \cdot B}\right)+T_{m}^{k}+U_{m, \tau}^{h} . \\
& =\Delta r^{k} \cdot 1_{m}^{k}+b_{m}-\Delta B^{k}+T_{m}^{k}+U_{m, \cdot}^{k} \cdot
\end{aligned}
$$

where

$\Delta r^{k}$ : difference of satellite true location, $r$ \& location given by navigation message

$r^{k, H}$

$1_{m}^{k}$ : Denotes the unit vector from the $k^{\text {th }}$ satellite towards the $m^{\text {th }}$ WRS.

$B^{k}$. is the true offset of the satellite transmission from GPS time.

$B^{k, B}$ : is the offset according to the navigation message.

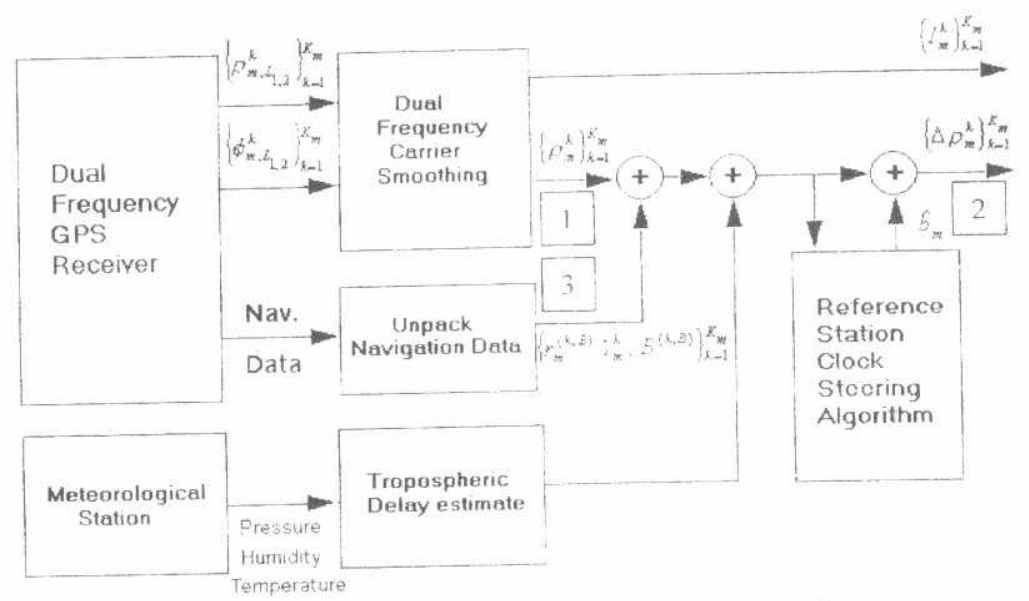

Fig.1: WRS with dual-frequency GPS receiver

WRS estimates the tropospheric delay on the basis of the elevation angle and local measurements of pressure, temperature and humidity. Indeed, a model without this auxiliary information can suffer errors of 2 meters for satellites at low elevation angles while with the surface measurements errors come down to about 30 centimeters.

Finally, the WRS uses a "clock steering" algorithm to estimate the WRS clock offset through averaging the reduced pseudorange of satellites in view. This averaging reduces the erroneous contribution due to the incividual satellite clock and ephemeris errors as well as the pseudorange measurement noise. In addition, if the WRS has a high quality clock such as a Rubidium oscillator, then the WRS clock estimates can be averaged over time to further reduce the clock estimate error.

The WRS clock estimates are subtracted from the smoothed pseudoranges to yield:

$$
\Delta p_{m}^{k}=\Delta r^{k} \cdot 1_{m}^{k}+\Delta b_{m}-\Delta B^{k}+T_{m}^{k}+t_{m i}^{k}
$$

As shown in Fig. 1, these pseudorange residuals are sent along with the vertical ionospheric delay estimates, $\left\{I_{m}^{k}\right\}_{n-1}^{k_{n}} ;$;o the WMS for further processing.

At the WRS site, a bit of information about the satellite ephemeris has to be communicated to the WMS. Three possible implementation issues are investigated 
- Transmission of satellite Pseudo Range Residuals (PRR : satellite ephemeris error, satellite clock error, receiver clock error and measurement noise :see 2 on Fig.1.) along with their confidence limits as well as the ionospheric delay estimates (See Eqn.5 ).This is the current implementation approach and will be referred to as the PRR approach.

- Transmission of the satellite Pseudo_Range(PR) along with the ionospheric delay estimates (See Eqn 3,1 on Fig. 1). This implementation approach is investigated and its pros and cons are clarified. It will be referred to as the PR approach.

- Transmission of the unit normals (Direction cosines for unit vector $1_{m}^{k}$ in Eqn 4 and 5) to the plane containing the WRS station, Earth center and each observed satellite by the given WRS along with the ionospheric delay estimates (See: 3 on Fig. 1). This implementation approach is proposed to avoid the cons of the other two approaches. Due to similarity to Space TRiangulation and being based upon angles adopted by Star TRackers, this will be referred to as the STR approach.

The wide area master station (WMS) should implement the proper hardware and algorithms (adapting to WRS implementation issue) for further processing of the relevant satellite information to yield the correction to be adhered to each satellite data.

\section{WIDE AREA MASTER STATION (WMS)}

The WMS incorporates two main estimators (see Fig.2 drawn for the PRR approach):

1. The orbit and clock error estimator (discussed in details hereafter).

2. The ionospheric delay estimator [7].

The former estimator is required to estimate both the ephemeris error and the clock error of the $\mathrm{j}$-th satellite. Three implementation approaches will be outlined in the following :

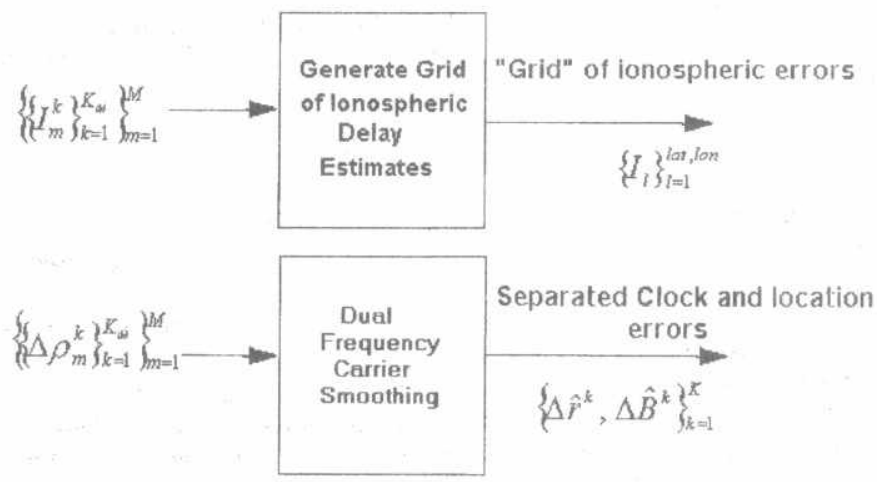

Fig.2. WMS (Residual-based) Estimators 


\section{1. PRR-based WMS Implementation}

On the basis of Eqn. 5, a least square (LS) algorithm is used in either of the following cases :

- Number of the WRS's seeing the satellite (number of measurements $m$ ) $=4$ :

Solution of Linear system of equation is immediately used

- Number of the WRS's seeing the satellite (number of measurements $m$ ) $>4$ :

Matrix Pseudo-inverse is used

- Number of the WRS's seeing the satellite (number of measurements $m$ ) $<4$ :

Minimum-Norm Algorithm (another matrix pseudo-inverse is used).

For evaluating the performance of such approach, data from the satellite tool kit [9](STK) and Klobachar model [17-19] for ionosphere delay error were used for orbit and clock error estimation. As performance indices, user position error and WMS processing latency have been evaluated over the Arab region for the ARWAAS system $[8,14,15]$. To evaluate the depth of coverage, accuracy for users near edges of the area of the network has been compared to accuracy at the center and found to degrade by a factor of 4 . As to the WMS processing latency, about 7 minutes have been required to generate the correction signal. Contrasting such latency to targeted time constraints defined by FAA in form of time-to-alarm (6-8 seconds) $[7,13]$, reveals the necessity to look forward for significant reduction of such processing latency.

\section{2. PR-based WMS Implementation}

Revisiting the original problem and formulation suggests the research reformulation of equivalent problems of orbit determination rather than orbit error determination. For a formulation on the basis of Eqn 3 , the following solutions are examined:

- Case-Switched Combined Direct Solution (DS) with LS solution (DS/LS).

- Case-Switched Combined DS with LS-replaced by DS-Based Iterative Solution (DS/DSBIS).

- Combined DS algorithm and DSBIS (DS-to-DSBIS)

- Orbit Propagator Solution (OPS).

III. 2. 1. Case-Switched Combined DS with LS Solution (DS/LS)

DS [16] has been applied to pseudo-range data to yield the satellite orbit in a noniterative approach and consequently reduces the processing time. DS is applied whenever the number of observations is exactly 4 while the LS is applied otherwise. With LS initial guess being selected as a point on the GPS satellite orbit, processing time comes down to about 120 seconds compared to 418 seconds under the same computing environment specifications given before. As to accuracy, no accuracy variation (from previously obtained) is experienced with this approach[12]

III. 2. 2. Case-Switched Combined DS with LS-replaced DS Based Iterative Solutions (DS/DSBIS)

An iterative algorithm based upon the DS is used to compute a least squares estimate of ephemeris error that is to be added to the initial guess and the final estimate at this given time will serve as the initial estimate at the next time. This algorithm is known to yield faster convergence rate compared to LS [16] and hence is particularly important in space missions or aircraft, where the initial guess may be 
far off from the real position. Comparison of the DS/DSBIS results to those given before shows that DS/DSBIS requires only 65 seconds as processing time at no accuracy expense

\section{2. 3. Combined DS and DSBIS (DS-to-DSBIS)}

This method depends on combining the DS with DSBIS, such that, the initial guess of the DSBIS is calculated from the DS. In other words, when the WRS's that see the satellite are more than four, then the DS selects randomly, any four WRS's to estimate the satellite position, and then, this estimation is used as initial guess for the DSBIS. DSBIS then iterate the solutions until obtain the required accuracy. The processing time cuts down to about 12 seconds under the same computing environment specifications given above and also at no accuracy penalty if not even better.

\section{2. 4. Orbit Propagator Solution (OPS)}

This solution derives the initial guess from combining the previous orbit estimate with an increment calculated from Kepler laws [1], for the satellite's orbital plane where the GPS follows a circular orbit. WMS calculates the changes in satellite velocity so as to predict changes in the position corresponding to the initial guess for the next measurements. Hence, the initial guess can be generated as follows:

$$
\boldsymbol{r}^{j}(\boldsymbol{n})=\boldsymbol{r}^{j}(\boldsymbol{n}-1)+\gamma \cdot \dot{\boldsymbol{r}}^{j}(\boldsymbol{n}-1)
$$

where $\quad r^{j}(n)$ : the initial guess for the satellite position in the instant $\mathrm{n}$.

$\boldsymbol{r}^{J}(\boldsymbol{n}-1)$ : the estimated satellite position in the instant $\mathrm{n}-1$.

$\gamma: \quad$ the propagation constant.

$\dot{r}^{\prime}(\boldsymbol{n}-1)$ : the orbital velocity $(3.8659 \mathrm{~km} / \mathrm{sec})$.

Two cases have been considered; namely: $\gamma=0$, and $\gamma=1$ yielding processing times of about $7.5 \mathrm{sec}$, and $6.5 \mathrm{sec}$ respectively.

\section{3. STR-based WMS Implementation}

Upon reception of each unit normal to corresponding planes (of given WRS station Earth center and each observed satellite by this WRS), a space triangulation problem is solved to yield the location of the observed satellite. The WRS share is implemented as follows :

- Unpack the navigation message of each satellite and get $\left(x_{s}, y_{s}, z_{s}\right)$; i.e. $r_{s}$

- Form $\left(x_{s}-x_{i}\right),\left(y_{s}-y_{i}\right),\left(z_{s}-z_{i}\right)$; hence get $r_{s i}$ direction

- Form the unit normal direction by the cross product $\left(r_{\mathrm{s}} \times r_{\mathrm{si}}\right)=\mathrm{n}_{\mathrm{si}}$

- Received set (minimum of 3 stations) of unit normal for a given satellite is used to get the location of this given satellite from the following equations :

$$
\left(r_{\mathrm{s}}-\mathrm{r}_{\mathrm{i}}\right) \cdot \mathrm{n}_{\mathrm{si}}=\mathbf{0}
$$

for WRS stations $i=1,2,3$ and satellites in view $s=1,2 \ldots$ etc. 
Inverting a matrix for each satellite in view solves this set of linear equations. Whenever the number of observing stations is larger than 3 pseudo-inverse is used and orbit propagator is then established.

\section{COMPARATIVE EVALUATION OF ORBIT ERROR ESTIMATION SOLUTIONS}

Results obtained for processing time as well as positioning errors induced by ephemeris error for the outlined methods are summarized in table 1. Results favor the OPS solutions on the bases of both accuracy and processing speed; this OPS is really the Kinematic approach to the solution of the ephemeris problem of the GPS system.

As to the WRS communicated data to WMS, the transmission of pseudo-range error evidently requires smaller word length compared to transmission of pseudo-range for comparable accuracy performance. The transmission of direction cosine exhibits a defined as well as naturally normalized dynamic range (values are always $<1$ ). Moreover. solution of the satellite location problem is naturally formalized in terms of linear system of equations allowing for more tangible and faster solution. Detailed MATLAB and STK results for this proposed STR approach will be given in a future paper.

Table 1: Accuracy and Processing Latency for Implemented WMS.

\begin{tabular}{|c|c|c|}
\hline $\begin{array}{c}\text { WMS Implemented } \\
\text { Solution }\end{array}$ & $\begin{array}{c}\text { RMS errors } \\
{[\mathbf{m}]}\end{array}$ & $\begin{array}{c}\text { Processing } \\
\text { Time [sec] }\end{array}$ \\
\hline Published Solutions & 1.9511 & 418 \\
\hline DS/LS & 1.943 & 120 \\
\hline DS/DSBIS & 1.866 & 65 \\
\hline DS-to-DSBIS & 0.9343 & 12 \\
\hline OPS $(\gamma=0)$ & 0.9544 & 7.5 \\
\hline OPS $(\gamma=1)$ & 0.81232 & 6.5 \\
\hline
\end{tabular}

\section{V.CONCLUSIONS AND FUTURE WORK}

The present work deals with WMS implementation covering the orbit and clock error estimation techniques on the bases of Kinematic, and Nondynamic approaches. Published approaches rely on solving the problem of orbit error estimation on the bases of transmitted pseudorange errors yielding inconvenient WMS processing latency. To reduce the processing time to a level conforming with the FAA time-toalarm requirement, this research has proposed to solve the equivalent problem of orbit determination rather than orbit error determination. This has permitted to find a direct solution instead of iterative solution used in the orbit error determination. Consequently, reformulation of the problem and implementation of the WRS have been elaborated. Research for accelerating and improving the performance of Nondynamic approach has resulted in reduction of processing time to about one fiftieth of the corresponding time obtained from direct programming of the published solutions. 
Suggestions for future research include improvement of WRS implementaion of single-site locator algorithm, WMS implementation of the dynamic techniques for satellite orbit and clock error estimation and use of tomographic technique for the ionospheric time delay errors correction

\section{REFERENCES}

[1] E. D. Kaplan, Ed., "Understanding GPS Principles and Applications," Norwood, Artech House, 1996.

[2] Y. C. Chao, "Real Time Implementation of the Wide Area Augmentation System for the Global Positioning System with an Emphasis on Ionospheric Modeling," PhD. Dissertation, Stanford University, June 1997.

[3] C. Kee, and B. Parkinson, "Wide Area Differential GPS as a Future Navigation System in the U. S.," Proceedings of IEEE PLANS'94, pp. 778-795.

[4] S. Peck, et.al., "WAAS Network Time Performance and Validation Results," Proceedings of ION-GPS 97, Institute of Navigation, 1997.

[5] J. Westbrook, "EGNOS: Introduction to system architecture, performance and Possibilities of Demonstration to potential user communities through the EGNOS Test Bed Program," Proceedings of Integration of LORAN-C/Eurofix and EGNOS /Galileo Conference, Bonn, Germany, March, 22-23, 2000.

[6] A. Shimamura, "MSAS (MTSAT Satellite-based Augmentation System) Project Status," Proceedings of GNSS 98, Toulouse, France, October, 20-23, 1998.

[7] P. Enge, el.al., "Wide Area Augmentation of the Global Positioning System," Proceedings of the IEEE, Vol. 84, No. 8, August 1996.

[8] S. Y. Issa, M. A. Matar, and A. M. Hamad, "ARWAAS Architecture and Implementation Issues," Proc. of the $8^{\text {th }}$ ASAT Conf., MTC, Cairo, 4-6 May, 1999.

[9] Satellite Tool Kits Software, Copyright 1989-1999 by Analysis Graphics, Inc.

[10] S. Pullen, P.K Enge and B.W. Parkinson, "A New Method for Coverage Prediction for the Wide Area Augmentation System (WAAS)," Proceedings of the Annual Meeting of the Institute of Navigation, Colorado Springs, June 1995.

[11] J. Ceva, et.al., "Incorporation of Orbital Dynamics to Improve Wide Area Differential GPS," Proc. of ION-GPS 95, Palm Springs, CA., Sept. 12-15, 1995.

[12] S. Y. Issa, "Satellite Navigation Systems: Accuracy Enhancement" PhD. Dissertation, Avionics Dept.,MTC ,Cairo, Egypt 2000

[13] J. M. Beukers, "Global Radionavigation - The Next 50 Years and Beyond," The Journal of Navigation, Vol. 53, No. 2, May 2000

[14] S. Y. Issa, M. A. Matar, and H. M. S. Abdel-Wahab, "Orbit and Clock Error Estimation for ARWAAS," Proc. of $8^{\text {th }}$ ASAT Conf., MTC, Cairo, 4-6 May, 1999.

[15] S. Y. Issa, and M. A. Matar, "Layout Issues for Implantation of the ARWAAS Reference Stations Network", Proc. of the $2^{\text {th }}$ EOEI Conf., Cairo, 11-14 Nov. 2000

[16] I. Biton, et.al., "Improved Direct Solution of the Global Positioning System Equation", J. Guidance, Control \& Dynamics, Vol. 21, No. 1, Jan.-Feb. 1998.

[17] W. A. Feess, and S. G. Stephens, "Evaluation of GPS lonospheric Time-Delay Model," IEEE Trans.Aerospace \& Electronic Sys., Vol. AES- 23, No. 3, May 1987

[18] D. Dai, et.al., "Optimal Use of Ionospheric Corrections for Wide Area Augmentation System (WAAS) Users," http://www. stanford edu/ dhdy/paper.

[19] J. Klobachar, "Ionospheric Time-Delay Algorithm for single Frequency GPS users," IEEE Trans. Aerospace \& Electronic Sys., Vol.AES-23, No. 3, May 1987. 